G211(P) TRANSFERRING THEM OUT WHILST THEY ARE STILL IN: THE EXPERIENCE OF THE EMERGENCY BED SERVICE WORKING WITH THE NEONATAL TRANSPORT TEAM

A Onibere, S Broster, S O'Hare, D Wari-Pepple, A Dhar. Acute Neonatal Transfer Service (ANTS), Cambridge University Hospital NHS Foundation Trust, Cambridge, UK

\subsection{6/archdischild-2018-rcpch.206}

Introduction The Acute Neonatal Transfer Service (ANTS) for the East of England is supported $24 / 7$ by Emergency Bed Service (EBS), whose role in recent years has extended to providing a cot location service for in-utero referrals from the 17 delivery units across the region. The process of locating both an antenatal bed and potential NICU cot is time-consuming involving multiple phone calls to several centres. Short-term outcomes of the process were reviewed.

Material and methods All in-utero referrals to the EBS from 1/ 1/2017-30/6/2017 were reviewed using the ANTS database. Demographic details and reasons for referral were identified. The outcome of the process including whether an antenatal transfer occurred, reasons for non-transfer, distance travelled for transfer and whether or not delivery occurred within 7 days was also recorded.

Results 193 in-utero referrals were made to EBS in the 6 month period, 41, 131 and 21 from Level 1, 2 and 3 units respectively. The 2 most common reasons for referral were a predicted need for a higher level of neonatal care $(n=99)$ and the regional NICU being closed $(n=82) .151$ of the women underwent antenatal transfer and of these, 51 delivered within 7 days. 112 were transferred to a centre within the East of England, 39 were sent out of region and this necessitated repatriation by ANTS back to the region. Of the 42 women who were not transferred, 8 declined, 29 too unstable to move to the identified receiving unit and in 5 , no bed/cot could be found. Amongst those transferred, 75 travelled less than 50 miles while 61 and 15 had to travel more than 50 miles and 100 miles respectively.

Conclusions $33.7 \%$ of infants referred in-utero delivered at the receiving centre within 7 days. $96 \%$ of these infants were extremely preterm ( $<27$ weeks). 50\% were transferred more than 51 miles away from their primary location needing retrieval by ANTS back to their local units. The emotional and financial impact on these families is yet to be explored. The Operational Delivery network is working on capacity planning to address capacity issues that resulted in $42 \%$ of referrals.

\section{G212(P) PREMEDICATION USE IN NEONATAL INTUBATION: ARE WE DENYING BABIES ADEQUATE ANALGESIA FOR INTUBATION?}

PRAM Paediatric Research Across the Midlands. Neonatal Sub Group, Paediatric Research Across the Midlands, Birmingham, UK

\subsection{6/archdischild-2018-repch.207}

Aims To assess the frequency of premedication use for neonatal unit intubations. To analyse medication use by condition of the baby and indication for intubation.

Methods Local guidelines for premedication use were reviewed. Data was collected contemporaneously in ten neonatal units (five ITU, four LNU, one SCBU) using a standardised proforma over an eight week period. Babies intubated on the unit were eligible for inclusion and were identified from weekly checks of the BadgerNet system and by reviewing the notes of ventilated babies. Data were collected on; weight, gestation, indication for intubation, choice and timing of medication, condition of the baby and documented reasons for not using premedication.

Results There were 307 intubations in total, 96 (31\%) occurred on labour ward and were excluded. 212 neonatal unit intubations were analysed. Sixty-six (31\%) of babies had no premedication for intubation. Of these; 30 (45\%) were classified as being spontaneously breathing, 21 (31\%) were apnoeic but could be mask ventilated, $6(9 \%)$ were difficult to mask ventilate and $4(6 \%)$ were apnoeic and bradycardic despite attempts to mask ventilate. An additional 12 (5\%) babies had unsuccessful intubation attempts without premedication followed by successful intubation with premedication. Forty percent of babies not receiving premedication had a documented reason for this decision in the notes. Reasons included; 'No IV access' (4), 'Unable to cannulate' (1) 'Difficult to mask ventilate' (3) and 'in/out surfactant' (6).

Units varied in their choice of medication. Morphine was used for analgesia in 55 intubations. Only three had a time gap of more than five minutes between morphine and musclerelaxant. Given the delayed onset of action of morphine most these babies are unlikely to have received adequate analgesia during intubation.

Conclusions Previous research demonstrates that premedication blunts the adverse physiological responses to neonatal intubation and improves the chances of successful intubation. Providing analgesia and sedation for intubation should be considered humane care. Our data demonstrates that premedication is not used in all non-emergency intubations and could be used more widely. These results could be used to change current practice leading to quality improvement in patient care.

Acknowledgements The main contributors to the project are $\mathrm{K}$ Atkinson, H Mc Dermott, H Vawda, K Harvey, A Henderson, D Bogue, L Duthie, O Kowobari, R Yew, J Brindley, G Bhat, $\mathrm{N}$ Kottayankandy and A Ratcliffe. Consultant members of PRAM provided advice and supported the project, in particular A Ewer.

\section{G213(P) FAMILY INTEGRATED CARE - IMPLEMENTATION IN A TERTIARY NEONATAL INTENSIVE CARE UNIT}

${ }^{1}$ MMN Mansour, 'RM Morris, 'S Davies, ' G Jones, 'A Lawes, 'S Edwards, 2J Perkins, ${ }^{2} \mathrm{C}$ Wyn, ${ }^{2} \mathrm{R}$ Evans. ${ }^{1}$ Neonatal Intensive Care Unit, Singleton Hospital, Abertawe Bro Morgannwg University Health Board, Swansea, UK; ${ }^{2}$ Volunteer Veteran Parent Group affiliated with Neonatal Intensive Care Unit, Singleton Hospital, Abertawe Bro Morgannwg University Health Board, Swansea, UK

\subsection{6/archdischild-2018-rcpch.208}

Aims In the Family-Integrated Care (FiCare) model in Neonatal Intensive Care Units (NICU), parents are primary care-givers, while nurses and doctors teach and coach them and experienced veteran parents provide peer support. FiCare was developed by the neonatal team at Mt Sinai Hospital, Toronto, who had been inspired by remarkably improved outcomes seen in Tallinn, Estonia. Inspired by this model, we undertook to develop and implement FiCare on our unit.

Method A proposal was prepared and discussed with the consultant and nursing leads. A multi-disciplinary team was quickly formed of enthusiastic nurses, parents, doctors, ANNPs and other members of staff. Planning meetings were regularly 
held to brainstorm ideas and to develop training materials. A WhatsApp group was initiated to maintain continuous links within this team. The four pillars of FiCare were addressed: Staff Education, Parent Education, NICU Environment and Psycho-Social Support.

After months of preparation and staff training, FiCare was launched at the end of October 2016. Step by step over the following months we implemented further aspects of our FiCare model. FiCare is established as standard care for all admissions to our NICU.

Results Badgernet was used to obtain outcome measures in two epochs: 1 st December 2015 - 1 st October 2016 (PreFiCare) and 1 st December 2016 - 1 st October 2017 (PostFiCare).

\begin{tabular}{lll} 
Abstract G213(P) Table 1 & & \\
\hline Outcome & $\begin{array}{l}\text { Pre-ficare } \\
\text { epoch }\end{array}$ & $\begin{array}{l}\text { Post-ficare } \\
\text { epoch }\end{array}$ \\
\hline NICU days receiving any of own mother's milk & $76 \%$ & $81 \%$ \\
NICU days receiving only own mother's milk & $56 \%$ & $63 \%$ \\
Babies born<33 weeks gestation discharged receiving any & $66 \%$ & $80 \%$ \\
of own mother's milk & & \\
Length of stay>90 th centile for UK, based on gestation & $6.3 \%$ & $3.9 \%$ \\
\hline
\end{tabular}

The results in table 1 also compare very favourably with our network, where to date, ours is the only unit to implement FiCare.

Conclusion Since FiCare launched, there has been an improvement in breast feeding rates and a reduction in length of stay. We have also noted a gradual cultural change on NICU, including increased awareness of developmentally appropriate practices and increased parents' readiness for discharge.

\section{G214(P) PRETERM INFANT TRANSFUSION SUPPORT: ADULT OR CORD BLOOD DONATION? A LONGITUDINAL STUDY OF CD71 EXPRESSION IN PRETERM, CORD AND ADULT BLOOD SAMPLES}

${ }^{1} \mathrm{M}$ Forrester, ${ }^{2} \mathrm{~S}$ Mohamed Cassim, ${ }^{3} \mathrm{~S}$ Armstrong-Fisher, ${ }^{1,3} \mathrm{M}$ Vickers. ${ }^{1} / \mathrm{mm}$ munology, University of Aberdeen, Aberdeen, UK; ${ }^{2}$ Neonatology, NHS Grampian, Aberdeen, UK; ${ }^{3}$ Blood Transfusion, Scottish National Blood Transfusion Service, Aberdeen, UK

\subsection{6/archdischild-2018-rcpch.209}

Background Several risk factors are implicated in the development of necrotizing enterocolitis (NEC), one of which is receiving a blood transfusion. Recent evidence suggests that newborn (cord) red blood cells expressing CD71 can suppress potentially damaging immune responses to bacteria colonising the gut. Therefore, a blood transfusion, currently from adult donors, might effectively dilute these preventative properties, resulting in damaging inflammatory responses against gut bacteria, and subsequent NEC.

Aim We wished to identify in neonatal cord blood samples the early red cell population $(\mathrm{CD} 71+/ \mathrm{CD} 235+)$ purportedly responsible for the immunosuppression, and to establish a time course of their development with respect to gestational age. We also compared to adult blood donors, and assessed their potential anti-inflammatory activity.
Method Residual routine blood samples were obtained from preterm babies in the Neonatal Unit, after informed parental consent. Sequential samples were obtained until the infants were discharged. Neonatal/cord/adult blood samples, were separated by density centrifugation into mononuclear (buffy) cell layer (MNC) and red cells (RBC). MNC and RBC populations were immunofluorescently labelled with anti-human CD71 and anti-human CD235a and analysed by flow cytometry. We have assessed normal umbilical cord (term, elective sections) and healthy adult blood to investigate the anti-inflammatory properties of newborn cord RBC compared to adult RBC in simulated in vitro transfusions. Pro- and anti-inflammatory cytokine production by innate immune cells in response to pathogenic gut bacteria were also evaluated.

Results The proportion of RBC and MNC CD71 + cells were significantly greater $(\mathrm{p}<0.0001)$ from neonates (term cord and preterm infants) compared to adults. While transfusion did elicit suppression of pro-inflammatory cytokine production in response to bacterial challenge, regardless of the donor $\mathrm{RBC}$ source, the net effect of adult RBC transfusion is dilution of the innate CD71 + population.

Conclusion Logistics aside, we suggest that transfusion using umbilical cord progenitor $\mathrm{RBC}$, rather than adult $\mathrm{RBC}$ donations, might afford an alternative option for pre-term infants to lessen their risk of post-transfusion associated NEC.

\section{G215(P) IMPROVING SAFETY AT THE VERY BEGINNING: A NOVEL NEONATAL DELIVERY SAFETY CHECKLIST}

AM Taylor, P Lewis, M Nugent, J Montague, S Mitra. Neonatal Unit, University College London Hospitals NHS Foundation Trust, London, UK

\subsection{6/archdischild-2018-rcpch.210}

Aim Resuscitation and stabilisation of newborn babies can be a complex process involving members of several multi-disciplinary teams. We aimed to improve early newborn care by reducing errors and improving safety at each stage of this process. The World Health Organisation (WHO) Surgical Safety Checklist ${ }^{1,2}$ has been shown to dramatically reduce morbidity and mortality in surgical patients. Using the same principles, we created a novel Neonatal Delivery Safety Checklist with an aim to improve multi-disciplinary communication and early care of the newborn.

Method We started by surveying all members of the multi-disciplinary team about their experiences of attending complex and preterm deliveries. We process mapped the stages involved in attending a delivery and combined this with the survey information. This formed the basis of the pilot project where, as in the WHO Surgical Safety Checklist, we identified three 'vital phases': a preparatory period; a pause before the delivery where the whole team is encouraged to answer the question 'are we ready?'; and before transfer to the neonatal unit. Results We piloted the checklist over two weeks and immediate feedback was gathered. A 'plan, do, study, act' cycle was then initiated to respond to feedback from the multi-disciplinary team. As a result, the Checklist has gone through seven variations to date to make it as user friendly as possible whilst retaining the key principles.

Admission temperature in babies born at $<32$ weeks has shown a dramatic improvement since The Checklist has been introduced for routine preterm deliveries. Nearly $50 \%$ of infants had admission temperature $<36.5^{\circ} \mathrm{C}$ in the month 